\title{
Aquaporin-4 antibody titration in NMO patients treated with rituximab
}

\author{
A retrospective study
}

OPEN

Paola Valentino, MSc

Fabiana Marnetto, MSc

Letizia Granieri, MSc

Marco Capobianco, MD

Antonio Bertolotto, MD

Correspondence to

P. Valentino:

paolaval81@hotmail.com
Supplemental data at Neurology.org/nn

\section{ABSTRACT}

Objective: We undertook an observational retrospective study to investigate the usefulness of aquaporin-4 (AQP4) antibodies (Ab) titration in the management of patients with neuromyelitis optica (NMO) treated with rituximab (RTX) by studying (1) the correlation between AQP4-Ab titer and disease activity, (2) the influence of RTX on antibody levels, and (3) the association between AQP4-Ab levels and responsiveness to RTX.

Methods: A cell-based assay was used for AQP4-Ab titration in 322 serum samples from 7 patients with NMO treated with RTX (median follow-up 65 months), according to a treatment-totarget approach. Serum samples were collected every month following standardized procedures.

Results: (1) In group analysis, AQP4-Ab titers correlated with the disease activity, showing higher titers during and preceding relapses than during remission. However, in individual analysis, an increase in AQP4-Ab titers and CD19+ B cells did not always precede a relapse. (2) A reduction of AQP4-Ab titers in the short-term and long-term period was observed during RTX treatment. (3) Reduction of AQP4-Ab titers was observed in responder patients both 3 months after RTX infusion and in the long-term follow-up. In one nonresponder patient, AQP4-Ab levels never decreased during the treatment period.

Conclusions: Titration of AQP4-Abs could be useful in the clinical management of patients with NMO treated with RTX: titration before each reinfusion and 3 months after each reinfusion may provide information about responsiveness to RTX. Although a relationship among AQP4-Ab levels, disease activity, and response to RTX was observed, the usefulness of AQP4-Ab titration to predict relapses is limited. Neurol Neuroimmunol Neuroinflamm 2017;4:e317; doi: 10.1212/ NXI.0000000000000317

\section{GLOSSARY}

Abs = antibodies; AQP4 = aquaporin-4; ARR = annual relapse rate; $\mathbf{C B A}=$ cell-based assay; $\mathbf{C R E S M}=$ Regional Referring Centre for Multiple Sclerosis; EDSS = Expanded Disability Status Scale; FC = flow cytometric; IFI = indirect immunofluorescence; $\mathbf{N M O}$ = neuromyelitis optica; $\mathbf{R T X}=$ rituximab.

Neuromyelitis optica (NMO) is a severe autoimmune disorder of the CNS. ${ }^{1,2}$ In the majority of cases, NMO is associated with the presence of autoantibodies to the water channel aquaporin- 4 (AQP4). ${ }^{3,4}$ AQP4 antibodies (Abs) have been proven to play a key role in the diagnosis and pathogenesis of $\mathrm{NMO},{ }^{5}$ and to predict a more severe course of the disease. ${ }^{6,7}$ However, the usefulness of longitudinal AQP4-Abs titer measurements to predict further relapses or as an indicator of rituximab (RTX) efficacy remains to be evaluated in actual clinical practice. ${ }^{8,9}$

Numerous studies have analyzed AQP4-Abs titers in relation to the stage of disease or during immunosuppressive therapies. ${ }^{8,10-17}$ Data so far have been inconclusive, due to numerous reasons, including the sensitivity of the method of titration, the duration of follow-up, the number of patients, and the number of samples collected. In our study, these parameters have been optimized, allowing the reliable evaluation of the effect of AQP4-Ab titers on disease

From Neuroscience Institute Cavalieri Ottolenghi (NICO) (P.V., F.M., L.G., A.B.) and Neurologia 2-CRESM (P.V., F.M., L.G., M.C., A.B.), AOU San Luigi Gonzaga, Orbassano, Turin, Italy.

Funding information and disclosures are provided at the end of the article. Go to Neurology.org/nn for full disclosure forms. The Article Processing Charge was paid by Fondazione per la Ricerca Biomedica.

This is an open access article distributed under the terms of the Creative Commons Attribution-NonCommercial-NoDerivatives License 4.0 (CC BY-NC-ND), which permits downloading and sharing the work provided it is properly cited. The work cannot be changed in any way or used commercially without permission from the journal. 

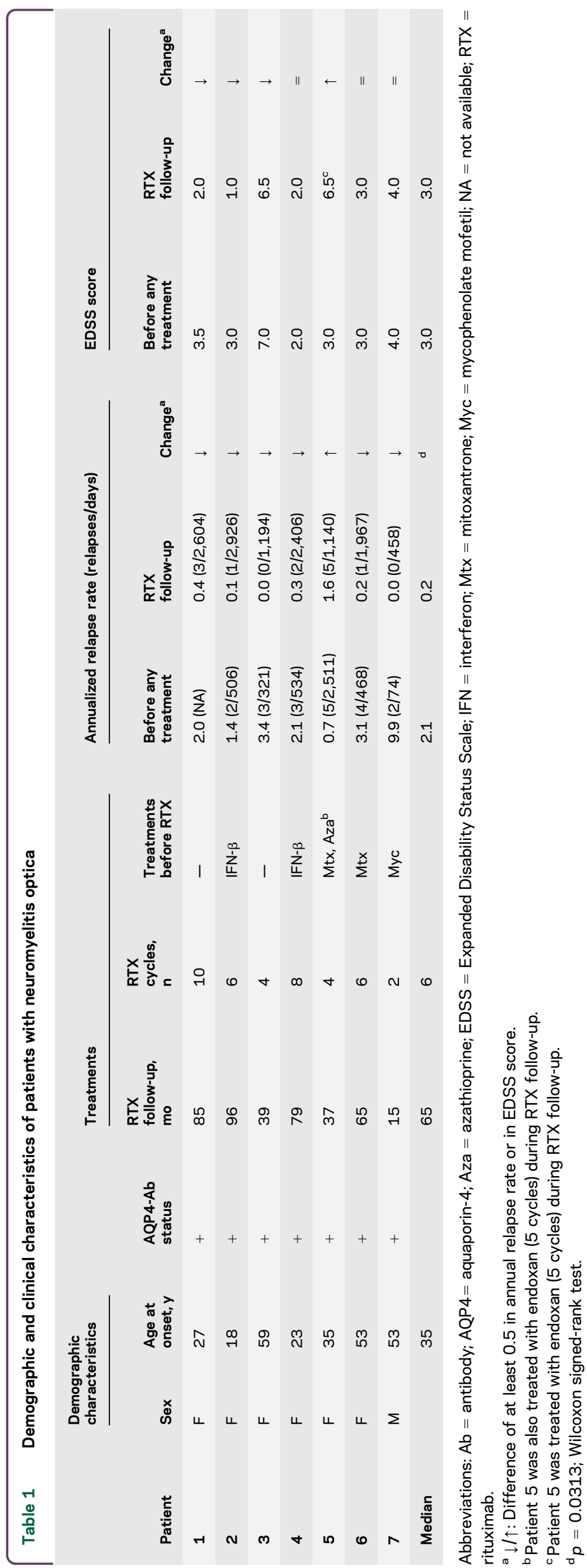

activity along with the efficacy of RTX, a monoclonal antibody considered to be one of the most efficient treatments of NMO. ${ }^{18-20}$

Our aim was to define the usefulness of AQP4-Ab titration in the clinical management of patients with NMO treated with RTX. In detail, we investigated (1) the association of AQP4-Abs titer with disease activity, (2) the effect of RTX therapy on AQP4-Abs levels, and (3) the association between responsiveness to RTX and change over time in AQP4-Ab titers.

METHODS Patients. This is an observational retrospective case series study, in which serum samples from $7 \mathrm{AQP} 4-\mathrm{Ab}-$ positive patients with $\mathrm{NMO}$ were evaluated for AQP4-Ab titer. Patients were diagnosed according to the 2006 Wingerchuk revised diagnostic criteria. ${ }^{2}$ The disease followed a relapsing course in all patients. Patients presented to the Regional Referring Centre for Multiple Sclerosis (CRESM) at Orbassano, Turin, Italy, for follow-up. Patient details are described in table 1.

All patients were treated with RTX and monitored following a treatment-to-target approach. Each patient started RTX therapy with RTX $375 \mathrm{mg} / \mathrm{m} 2$ once a week for 4 weeks, while the subsequent RTX cycles $(1,000 \mathrm{mg}$ infused twice, with a 2 -week interval) were given whenever the percentage of $\mathrm{CD} 19+\mathrm{B}$ cells was more than $0.1 \%$ in peripheral blood mononuclear cells. ${ }^{21-23}$ Details of the treatments used by patients before RTX are described in table 1. Treatment regimens during clinical relapses included IV methylprednisolone (1,000 $\mathrm{mg}$ for 5 consecutive days without tapering) and oral prednisone (25 mg for 10 days) (figure 1 ).

The median follow-up of RTX treatment in the present study was 65 months (range 16-96) for a total of 417 months of RTX follow-up. Four patients were followed for at least 60 months. Forty total RTX infusions were administered (median 6 infusions/patient; range 2-10 infusions/patient). The median interval between treatments was 11.0 months (range 4.0-36.3).

A blood sample was collected every month and serum samples were stored at $-80^{\circ} \mathrm{C}$ in the CRESM biobank until AQP4-Ab detection.

Standard protocol approvals, registrations, and patient consents. The use of blood samples from the CRESM biobank was approved by the ethical committee of AOU San Luigi Gonzaga (approval 7777/2013). All patients provided written informed consent for the use of their blood banked samples.

Indirect immunofluorescence (IFI) cell-based assay (CBA) for AQP4-Ab detection. AQP4-Abs were assessed using a CBA based on IFI (FA 1128-1010-50; Euroimmun, Lubeck, Germany), according to manufacturer's instructions. IFI reactions were analyzed using Nikon (Tokyo, Japan) Eclipse $90 \mathrm{i}$ with a $\times 20$ magnification. Positive and negative human control sera (Euroimmun) were tested in each working session. ${ }^{24}$

Samples were titrated using serial twofold dilutions from 1:80 to $1: 5,120$. The titer was defined as the sample dilution factor for which specific fluorescence was barely but clearly identifiable. Antibody titer was expressed as the corresponding dilution factor.

The increase of AQP4-Ab level was empirically defined as a titer rise at least 2-fold higher than the last previous lowest value. A total of 1,340 IFI reactions were performed. Two different 
A. Patient 1

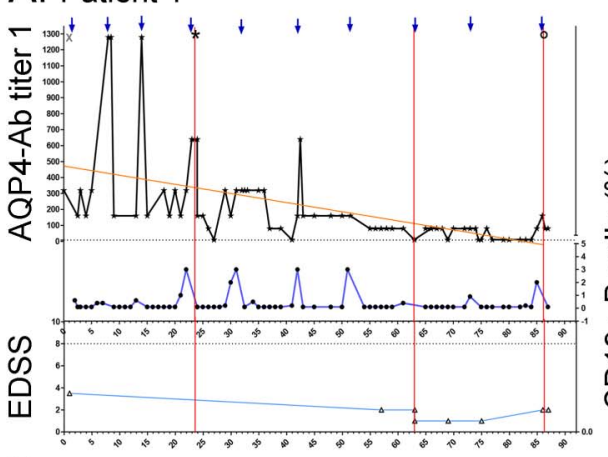

C. Patient 3

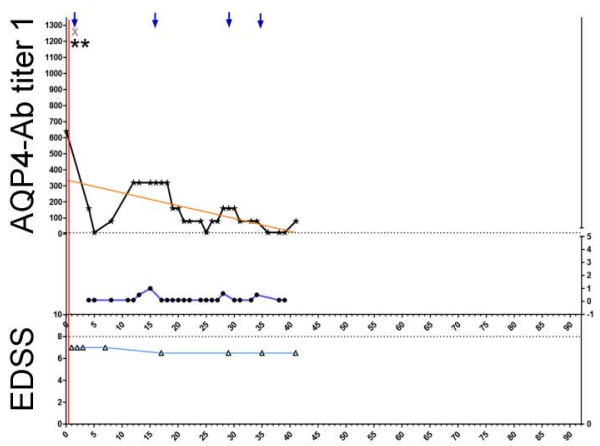

E. Patient 5

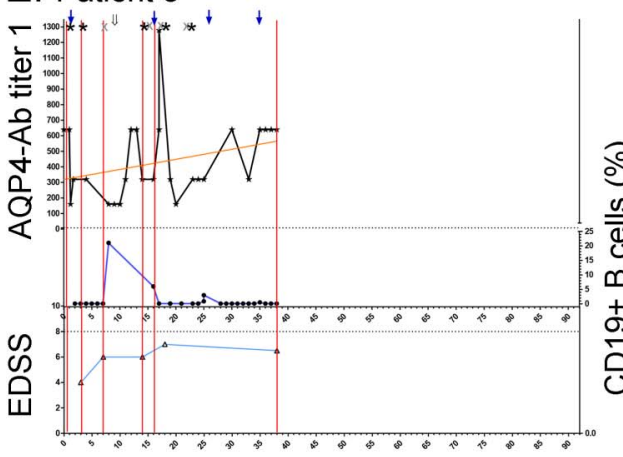

G. Patient 7

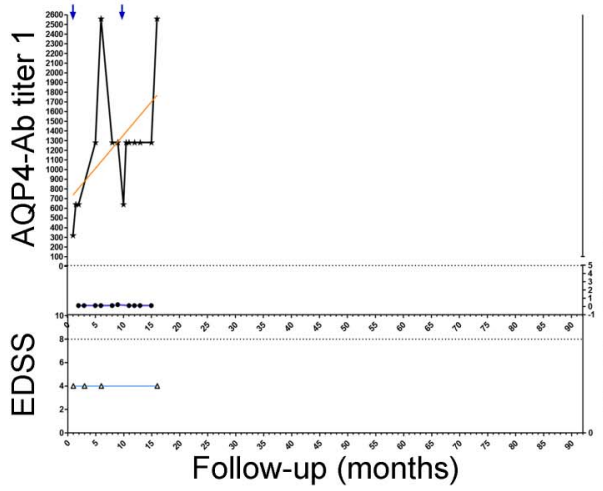

B. Patient 2

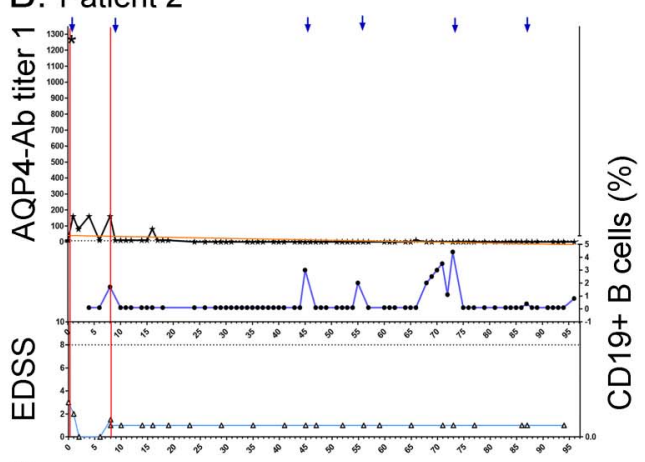

D. Patient 4

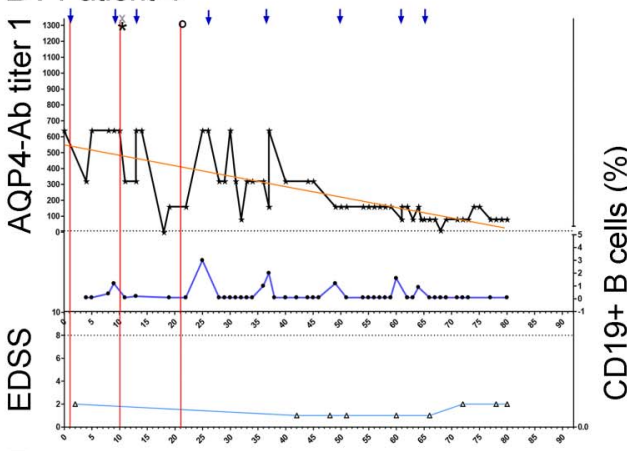

F. Patient 6

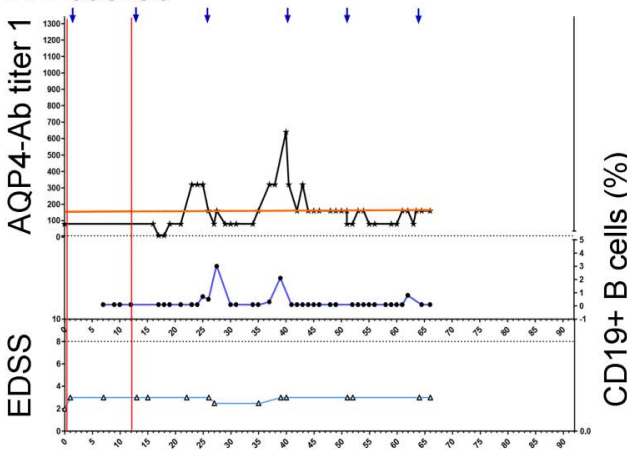

Follow-up (months)

- AQP4-Ab titer

- AQP4-Ab trend

- CD19+ B cell count

- EDSS

I Relapse

$\downarrow$ RTX

$\Downarrow$ Endoxan

* Methylprednisolone

- Prednisone

$x$ Plasma exchange

(A-G) Relationship among AQP4-Ab serum levels, CD19+ B cell percentage, Expanded Disability Status Scale (EDSS) score, relapses, and treatments in 7 patients with neuromyelitis optica. Patient follow-up was analyzed since the first RTX infusion (month 1). AQP4-Ab titers were expressed as the corresponding dilution factor.

operators (F.M. and L.G) independently evaluated 150 reactions to assess the intralaboratory and interrater reproducibility of the assay. The operators were also blind to the sample type. Euroimmun provided all the kits.
Flow cytometric (FC) analysis for CD19+ B-cell detection. The percentage of CD19+ B cells was evaluated by FC on the blood samples collected monthly in ethylenediaminetetraacetic acid tubes, using antibodies directed against CD45/CD20/CD19 
(Becton Dickinson, San Jose, CA). FC data acquisition was performed on a BD FACSCanto II (Becton Dickinson). B cells were identified as CD19+CD20+ cells. The data acquired were analyzed using BD FACS DIVA Software (Becton Dickinson). Instances that reported observed value of $<0.1 \%$ of total lymphocytes were defined as undetectable levels of $\mathrm{CD} 19+\mathrm{CD} 20+$ immunophenotype. ${ }^{23}$

Clinical assessment. Since there are no guidelines to define RTX responder and nonresponder patients, we tried to establish selection criteria based on a routine clinical parameter to classify our patients. In particular, we utilized the Expanded Disability Status Scale (EDSS) and the annual relapse rate (ARR). In addition, we defined an exclusion criterion based on follow-up period of less than 2 years considering the nature of the disease and the treatment. ARR and EDSS score were evaluated for each patient during the follow-up. Clinical relapses were defined as patientreported or objectively observed events typical of an acute inflammatory demyelinating event in the CNS, with duration of at least 24 hours, in the absence of fever or infection, documented by contemporaneous neurologic examination. Disability was evaluated according to EDSS during stability periods.

Statistical analysis. Statistical analysis was performed using GraphPad (La Jolla, CA) PRISM 5. In particular, the following nonparametric tests were used as appropriate to analyze AQP4$\mathrm{Ab}$ titers and clinical features: Mann-Whitney test, KruskalWallis test, Wilcoxon matched-pairs test, Friedman test, Fisher exact test, Spearman correlation test, and 2-way analyses of variance. $p$ Values $<0.05$ were considered statistically significant.

Reproducibility of the assay was evaluated by the interrater agreement kappa test. ${ }^{25,26}$

RESULTS AQP4-Ab titer was evaluated in 322 serum samples (median 44 samples/patient; range 16-75 samples/patient) from 7 patients (figure 1). Median AQP4-Ab titer was 160 (range 0-2,560). A total of 264 out of the total 322 samples tested positive for AQP4-Abs (median 160; range 10 2,560).

A total of 150 samples were evaluated in a blinded study by 2 operators. A $100 \%$ interrater $(\kappa=1)$ and a $100 \%$ interrun agreement $(\kappa=1)$ was found. In the present study, grouped analysis and single timepoint (individual) analysis were performed to study fluctuations of AQP4-Ab levels according to disease activity and to RTX treatment. In addition, clinical usefulness of AQP4-Ab titration was investigated by evaluating responsiveness to RTX.

Do AQP4-Ab titers correlate with clinical disease activity? Using group analysis, AQP4-Abs were determined in all available samples $(n=10)$ from 5 patients at onset of relapse. These samples showed higher median AQP4-Ab levels (median titer 320; range 160-640) than samples collected during remission $(\mathrm{n}=261$; median titer 80; range $0-1,280)(p=0.0002$, MannWhitney test). Moreover, the analysis of the samples collected within 3 months prior to onset of relapse ( $\mathrm{n}=23$; median titer 320 ; range $0-640$ ) also demonstrated the same difference (figure 2A). Higher AQP4-Ab levels were observed during
Figure 2 Aquaporin-4 (AQP4) antibody (Ab) titers during remission and relapse

A

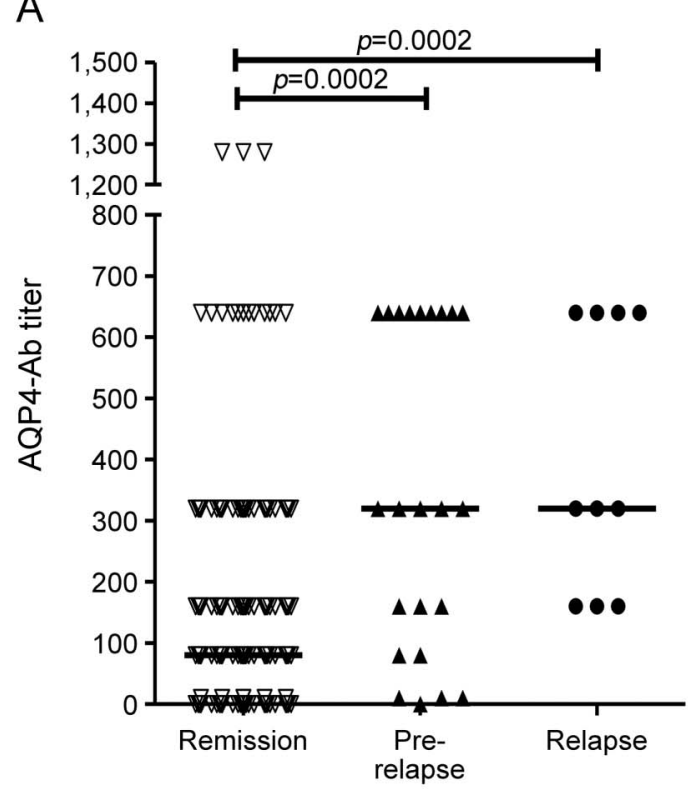

B

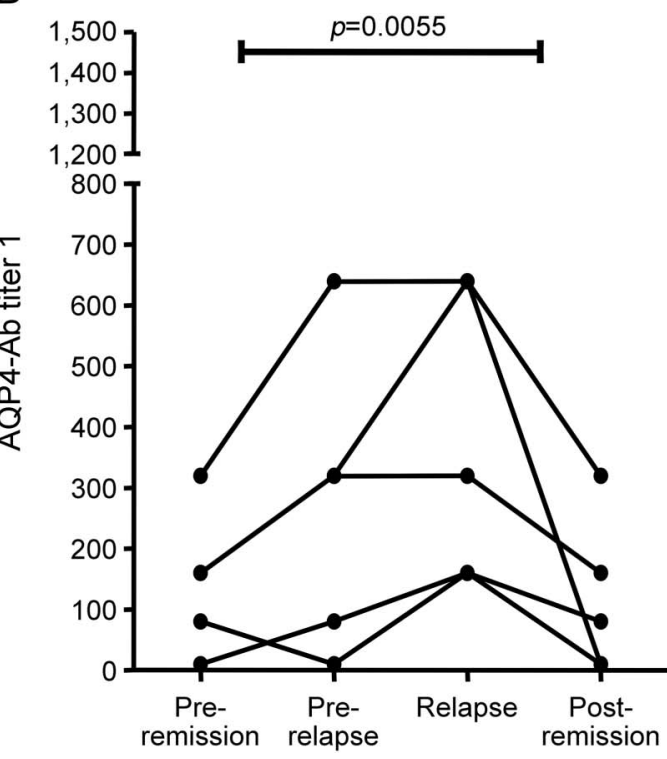

AQP4-Ab levels were correlated to disease activity. (A) Median AQP4-Ab titers were higher $(p=0.0002$; MannWhitney test) during relapse ( $\mathrm{n}=10$; median titer 320 ; range 160-640) and within 3 months before relapse $(n=$ 23; median titer 320; range 0-640) compared with remission period ( $n=261$; median titer 80 ; range $0-1,280$ ). (B) AQP4-Ab levels were compared in all available matched samples $(n=5)$ corresponding to 4 time points for each single relapse: this analysis was performed in order to avoid the bias caused by the unbalanced numbers of samples in the group analysis. These points are (1) remission before relapse (4-6 months preceding relapse), (2) prerelapse time (1-3 months preceding relapse), (3) onset of relapse, and (4) remission postrelapse (2-6 months after relapse). AQP4-Ab levels were confirmed to be higher during and preceding relapses than during remission $(p=0.0055 ;$ Friedman test). 
and preceding relapses than during remission in matched samples $(\mathrm{n}=5 ; p=0.0055$; Friedman test) (figure 2B).

Each single event of an increase in AQP4-Ab titers was studied by assessing the correlation with clinical disease activity and CD19+ B cells during RTX treatment (individual analysis). The aim was to establish if there is a correlation between AQP4-Ab titers and CD19+ B cells and if one or both variables could represent a predictive marker of relapse.

Increases in AQP4-Ab levels showed a weak association with clinical relapses and CD19+ B-cell recurrence. Similarly, no association was found between CD19+ B-cell recurrence and relapses (table 2). Finally, no correlation was found between $\mathrm{CD} 19+\mathrm{B}$-cell counts and AQP4-Ab titers $(p=0.0612$, Spearman correlation; figure e-1 at Neurology.org/nn).

Does RTX treatment influence AQP4-Ab levels? AQP4Abs were evaluated during RTX treatment $(\mathrm{n}=316$ samples; median titer 160; range 0-2,560). AQP4Abs were detectable in 258 out of the total 316 (82\%) samples. One patient (patient 2) seroconverted since the 25th month of follow-up. In the treatment-totarget approach, RTX treatment is monitored using CD19+ B-cell count: RTX infusion causes CD19+ B-cell decline $(<0.1 \%)$, and CD19 reappearance is considered to be an indication for RTX reinfusion. ${ }^{23}$

AQP4-Ab levels were reduced in samples collected at least 30 days after RTX infusion as compared to samples at the timepoint of RTX infusion ( $p=0.0015$, Mann-Whitney test; figure 3A). It is noteworthy that at the postinfusion timepoint the $\mathrm{CD} 19+\mathrm{B}$ cells were undetectable, while at the time of infusion, the CD19+ B cells were $>0.1 \%$. The first cycle of RTX was never included in the analysis, because data on $\mathrm{CD} 19+\mathrm{B}$ cells were unavailable.

Further, the short-term and long-term effects of RTX treatment on AQP4-Ab levels were investigated.

Short-term effects. AQP4-Ab levels were evaluated in all available paired samples $(\mathrm{n}=28)$ at 2 specific timepoints, namely, during RTX reinfusion and 3 months after (figures $3 \mathrm{~B}$ and e-2), showing a reduction in antibody titers $(p=0.0012$, Wilcoxon matched-pairs test).

Long-term effects. AQP4-Ab serum levels were also evaluated during a prolonged RTX follow-up (range 1-72 months), showing a reduction in annual median titer $(p<0.0001$; Kruskal-Wallis test). This effect was particularly marked after the first 2 years of therapy ( $p=0.0014$; Mann-Whitney test) (figures $3 \mathrm{C}$ and e-3).

Are AQP4-Ab titers associated with RTX responsiveness? Responsiveness to RTX was evaluated in patients with at least a 2-year follow-up, by the assessment of clinical parameters such as the ARR and the EDSS score (table 1).

The median ARR of patients was reduced at the end of RTX follow-up compared to pretreatment time $(p=0.0313$; Wilcoxon signed-rank test). In particular, 6 out of the 7 patients (86\%) showed a marked reduction of ARR during RTX follow-up.

During RTX follow-up, a total of 12 relapses occurred (median 1 relapse/patient; range 0-5). Seven relapses occurred in responder patients (median 1 relapse/patient) and 5 in the nonresponder patients.

\begin{tabular}{|c|c|c|c|c|c|c|c|c|c|c|}
\hline Table 2 & n between & quaporin-4 & ) antibody & (b) in & ease, clinic & relapses, & CD19+ B-c & recur & nce & \\
\hline Patients & \multicolumn{3}{|c|}{ Clinical relapses } & \multicolumn{4}{|c|}{ AQP4 increases } & \multicolumn{3}{|c|}{ CD19 increases } \\
\hline All patients $(n=7)$ & 12 & $5 / 11(45)$ & $7(58)$ & 24 & $15(63)$ & $5(21)$ & 7 (29) & 31 & $15(48)$ & 7 (23) \\
\hline Nonresponder $(n=1)$ & 5 & $1 / 5(20)$ & $1(20)$ & 2 & $0(0)$ & $1(50)$ & $1(50)$ & 4 & $0(0)$ & $2(50)$ \\
\hline Patient 1 & 3 & $2 / 3(67)$ & $3(100)$ & 8 & $7(88)$ & $2(25)$ & $1(13)$ & 9 & 7 (78) & 3 (33) \\
\hline Patient 2 & 1 & $1 / 1(100)$ & $1(100)$ & 3 & $2(67)$ & 1 (33) & 1 (33) & 5 & $2(40)$ & $1(20)$ \\
\hline Patient 3 & 0 & - & - & 2 & $2(100)$ & $0(0)$ & $0(0)$ & 3 & $2(67)$ & $0(0)$ \\
\hline Patient 7 & 0 & - & - & 2 & $0(0)$ & $0(0)$ & $2(100)$ & 1 & $0(0)$ & $0(0)$ \\
\hline
\end{tabular}

Abbreviation: $\mathrm{NA}=$ not available.

${ }^{\text {a }}$ Percentage values are calculated based on availability of AQP4-Ab data.

${ }^{b}$ Percentage values are calculated on the total number of relapses (all CD19+ B-cell data were available). 
A

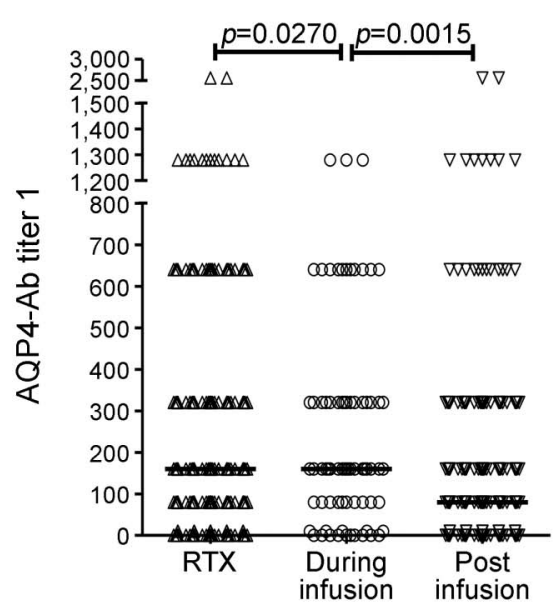

B

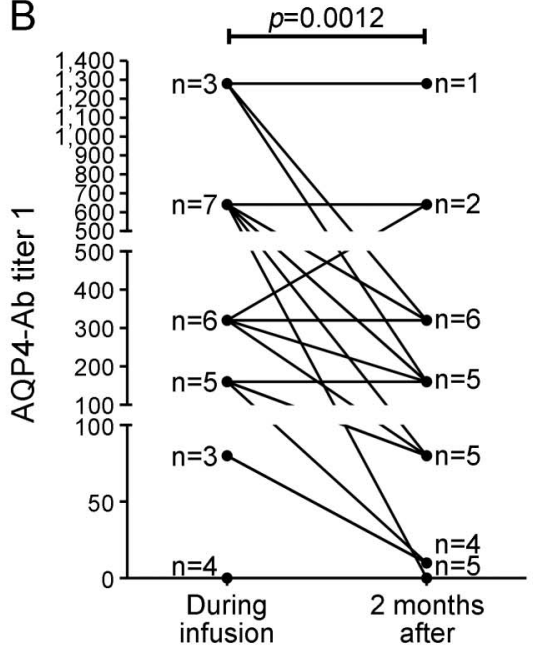

C

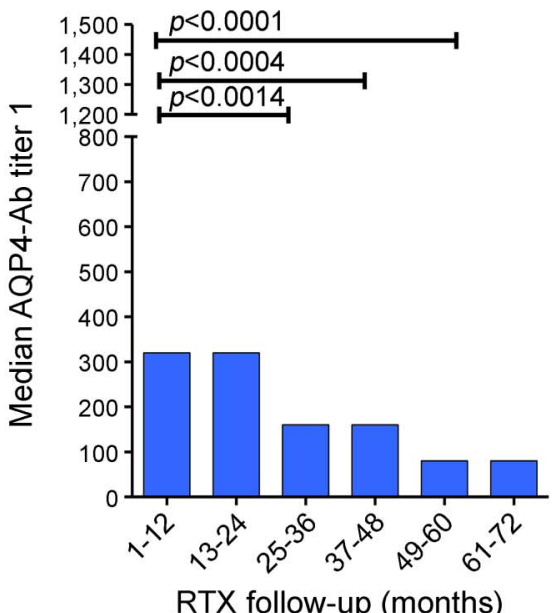

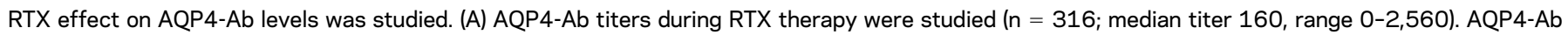

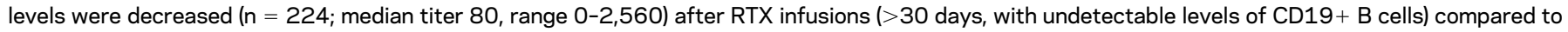

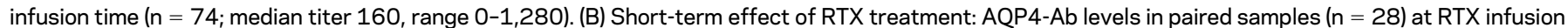

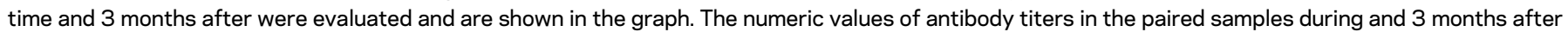

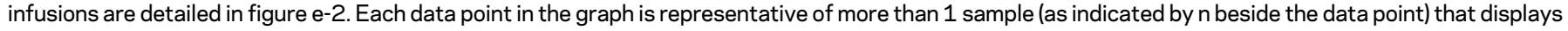

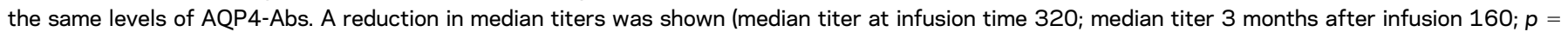

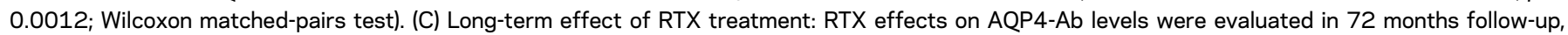
showing a decrease in median annual titers ( $p<0.0001$; Kruskal-Wallis test).

Two patients (29\%) were relapse-free during RTX follow-up.

The EDSS score improved or stabilized in 6 patients.

One patient (patient 5) showed a poor clinical response: the ARR increased and a worsening of EDSS score was observed, despite RTX treatment. In this patient, RTX was discontinued after 34 months of treatment.

Based on clinical parameters, patients 1, 2, 3, 4, and 6 were defined as responders, while patient 5 was defined as a nonresponder. Patient 7 was not included in further analysis due to insufficient RTX follow-up (15 months) for evaluation of responsiveness. Next, the short-term and long-term association of RTX responsiveness to AQP4-Ab titers was investigated (figure 4).

Short-term effects. The effect of RTX in responder and nonresponder patients at 3 months postinfusion was evaluated in all available paired samples $(n=27$; figures $4 \mathrm{~A}$ and e-2), showing a different distribution between responder and nonresponder groups. ( $p=$ 0.0410; Fisher exact test). In responder patients, the effect of RTX in reducing AQP4-Ab levels was stronger than observed while considering all the patients ( $p=0.0003$; Wilcoxon matched-pairs test).

Interestingly, upon the exclusive analysis of AQP4Abs after the first 2 RTX reinfusions $(n=11)$, the reductions in antibodies titer were reported only from responder patients $(\mathrm{n}=8)$. In the nonresponder patients, increased or steady titers were observed $(n=2$; $p=0.0545$; Fisher exact test) (figure e-2).

Long-term effects. In the long-term follow-up, an alteration in median AQP4-Ab annual levels was observed depending upon the responsiveness to RTX (figures 4B and e-3). This difference was stronger since the second year of therapy $(p<0.0001$ for responsiveness to RTX treatment, 2-way analysis of variance). In particular, AQP4-Ab levels decreased in 4 patients (patients 1, 2, 3, 4), but increased in the nonresponder patient 5. Patient 6 did not show a change in AQP4-Ab levels during his RTX follow-up (figures $4 \mathrm{C}$ and e-3).

DISCUSSION Detection of AQP4-Abs has been included in the NMO diagnostic criteria since 2006. ${ }^{2}$ The pathogenic role of these antibodies has been suggested in several studies. ${ }^{13,27-29}$ However, it is debated whether AQP4-Ab serum concentration is related to disease activity and, thus, whether its assessment could be helpful in predicting disease evolution and adapting the ideal treatment. ${ }^{8}$

Numerous studies have analyzed the correlation between AQP4-Ab titers and the stage of disease or the effectiveness of immunosuppressive therapy, ${ }^{8-17}$ with conflicting results.

The conflicting data could be attributed to numerous reasons: (1) methods used for titration, ${ }^{5,10-17}$ (2) 


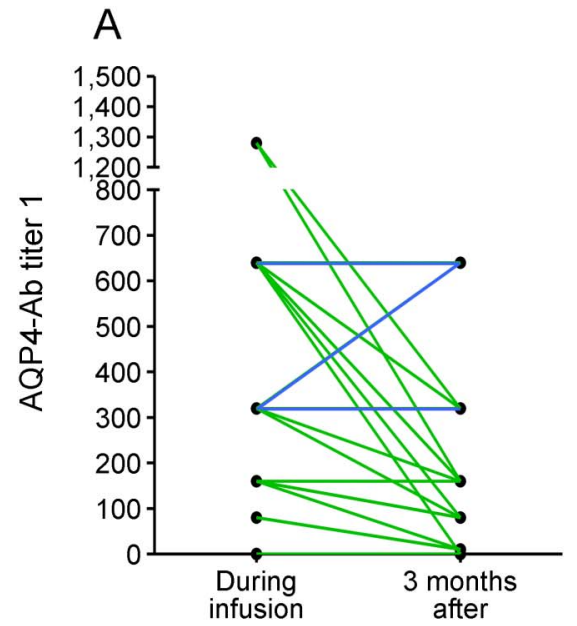

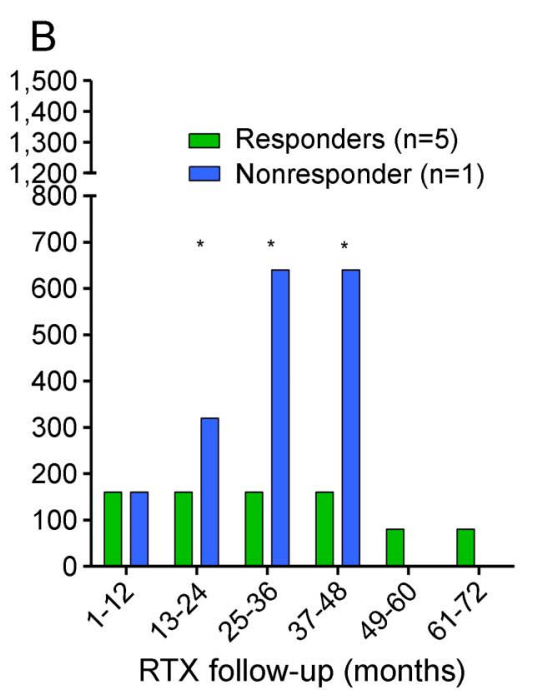

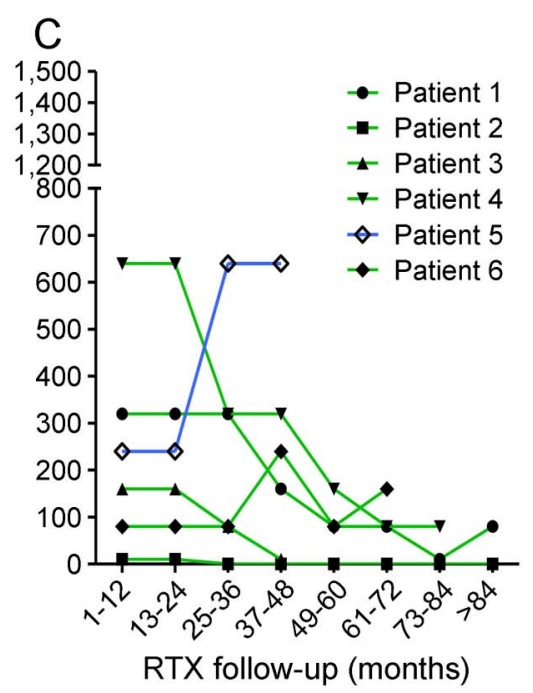

AQP4-Ab titer was evaluated according to RTX responsiveness in grouped or single patients. (A) Short-term effects of RTX treatment: RTX infusions cause a strong reduction in AQP4-Ab levels 3 months after RTX infusion only in responder patients (green lines, $n=24 ; p=0.0003$, Wilcoxon matched-pairs test). In the nonresponder patient (blue lines, $n=3$ ), AQP4-Ab levels were stable or increased after RTX infusion. (B) Long-term effects of RTX treatment: a difference was shown between responders and nonresponders since the second year of therapy (2-way analysis of variance, responsiveness to RTX treatment $p<0.0001$ ). (C) The alteration in AQP4-Ab levels was analyzed for each patient, showing that median annual AQP4-Ab titer decreased in 4/5 responder patients (patients 1, 2, 3, 4) during their whole follow-up (green lines), while, in nonresponder patient 5 (blue line), titer increased; patient 6 did not show a change in AQP4-Ab levels during his RTX follow-up.

length of the follow-up period (19-62 months), (3) number of patients (3-39 patients), (4) number of tested samples (26-601 samples; median 3-15 samples/ patient) and collection timing, and (5) number of RTX cycles (1-110; median 1-4 cycles/patient).

The wide range of variability observed in these parameters across the literature ${ }^{8,10-17}$ could at least partially explain the lack of consistency between results obtained and hamper comparison between different studies. In our study, these features have been taken care of to the best possible extent as discussed in detail in the previous sections.

We aimed to define the usefulness of longitudinal assessment of AQP4-Ab titration in the clinical management of patients with NMO treated with RTX. Results obtained in the group analysis show a general increase in $\mathrm{AQP} 4-\mathrm{Ab}$ titer during and, interestingly, also preceding attacks, in agreement with data obtained in some of the previous studies. ${ }^{12-15}$ However, high levels of antibodies did not always lead to a clinical relapse in individual patients, and the association varied widely (table 2). Thus, according to our data, AQP4-Abs cannot be used as specific markers for predicting an oncoming relapse. This may be because AQP4-Abs do not represent the sole cause of NMO disease. Other factors, such as blood-brain barrier damage, cytokine profiles, or T-cell activation, may play a role as well. ${ }^{10,14}$

The correlation of antibodies with clinical disease activity and the effects of RTX treatment on antibody levels, however, suggest the clinical and immunopathogenic importance of AQP4-Ab titers in management of NMO.

In our study, patients were treated with RTX, according to a treatment-to-target protocol. ${ }^{23}$ During RTX therapy, a reduction of AQP4-Ab titers in both the short-term (3 months after infusion) and longterm period (72 months follow-up) was observed. The spontaneous course of NMO spectrum disorder in untreated patients with respect to the levels of AQP4 antibodies is unknown considering it is unethical to deny treatment to any diagnosed patient. There is no evidence from the literature about a spontaneous reduction of AQP4-Ab levels. In fact, there is evidence available to indicate that the presence of AQP4-Abs can be detected as early as a decade before the onset of the disease. ${ }^{30,31}$ Therefore, there is a general consensus that the reduction of the autoantibodies is most likely mediated by the treatment regimen. ${ }^{32}$ As described by other studies, ${ }^{10,11,14}$ the decrement of AQP4-Ab levels may be due to the mechanism of action of RTX, which may lead over time to decreased production of immunoglobulins $\mathrm{G}$ and $\mathrm{M}$. This may predispose the patient to hypogammaglobulinemia and associated infections. ${ }^{33,34}$ To date, in our cohort, patient 2 , who is the only patient who seroconverted after RTX, recently developed hypogammaglobulinemia. Our experience suggests that although decrement of the pathogenic antibodies is an important goal in NMO management, caution 
needs to be exercised to ensure patient well-being in the long term.

An interesting correlation between AQP4-Ab levels and RTX responsiveness was observed. Reduction of AQP4-Ab levels induced by RTX short-term and long-term effects were observed exclusively in responder patients. In particular, AQP4-Ab reduction occurred in responder patients as early as after the first 2 RTX reinfusion cycles (observed at 3rd month after each infusion); increased or steady titers were observed in the nonresponder patient. In the longterm follow-up, a change in AQP4-Ab levels was observed depending upon the treatment responsiveness since the second year of therapy, with a decreasing titer observed in responder patients.

These findings, if confirmed, could be of great clinical relevance in patient management, allowing early identification of responder patients along with clinical evaluation. In our experience, a responder patient seems to be characterized by 2 relapses or less in the first 2 years of treatment and by a reduction of AQP4-Ab titer 3 months after each RTX infusion and during the longterm follow-up. These data suggest a possible strategy for identification of RTX responder patients within the first RTX cycles, by titrating AQP4-Ab during RTX reinfusion and after 3 months.

As per standard clinical practice, our patients were monitored for CD19+ B cells to determine the RTX reinfusion timing. The median time to $\mathrm{B}$-cell repletion in our patients was 11 months. In addition, 5 out of the 7 patients relapsed while technically using RTX: this may suggest that the FC method is not sensitive enough to consider CD19+ B cells a reliable marker predictive of relapses or of RTX responsiveness (table 2). The finding of new markers (i.e., $\mathrm{CD} 27^{10}$ ) or more sensitive techniques (i.e., $\mathrm{PCR}^{23}$ ) may present a solution to this problem. A poor association was shown between AQP4-Ab increase and $\mathrm{CD} 19+\mathrm{B}$-cell recurrence. Patients still tested positive for AQP4-Abs despite CD19+ B-cell percentage being undetectable in $78 \%$ of tested samples. Moreover, no correlation between CD19 counts and AQP4-Ab values was observed (figure e-1). These findings, observed in other studies as well, ${ }^{10,14}$ could be attributed to the mechanism of action of RTX, which depletes peripheral B cells without affecting plasma cells.

Some limitations of this study include the number of patients analyzed; in particular, the single nonresponder patient found in our study limits evaluations about RTX responsiveness. Observations about AQP4-Ab levels during relapse and remission could have been partially masked by RTX treatment. However, AQP4-Ab titers cannot be evaluated in untreated patients due to ethical constraints, as useful treatments are available.
On the other hand, the high number of samples studied, the careful and standardized procedure of monthly sample collection, processing, and storage, the long follow-up under RTX treatment, and the availability of precise clinical data represent the robustness of data obtained in the present study.

Our study shows a correlation between AQP4-Ab levels and clinical attacks in grouped analysis. We observed higher levels of antibodies during and preceding relapses. However, this correlation is lost upon a detailed individual analysis. We also observed a reduction in AQP4-Ab levels during RTX therapy in both the short and the long term. We report an alteration in AQP4-Ab levels according to treatment responsiveness. In particular, the reduction of AQP4-Ab titer 3 months after each RTX reinfusion observed in responder patients even in the initial RTX cycles could suggest a role for AQP4-Ab titration as biological tool to identify responder patients, with the support of clinical evaluations. Therefore, these findings merit further studies for validation of AQP4-Ab titration as a marker of responsiveness.

\section{AUTHOR CONTRIBUTIONS}

A.B., F.M., and L.G. conceived and coordinated the study. P.V., F.M., and L.G. collected and processed the samples and performed the laboratory analyses. P.V. and F.M. performed interpretation of the data and wrote the manuscript. P.V. performed statistical analyses. A.B. and M.C. were responsible for patient care and clinical documentation. All authors contributed to the critical review of the manuscript. All authors read and approved the final version of the manuscript.

\section{ACKNOWLEDGMENT}

The authors thank Euroimmun Italia and Euroimmun (Lübeck, Germany) for providing all kits (FA 1,128-1,010-50) and control samples and Ishira Nanavaty for linguistic assistance.

\section{STUDY FUNDING}

This study was supported by Fondazione Italiana Sclerosi Multipla, FISM (2014/PMS/1), and Fondazione Ricerca Biomedica Onlus.

\section{DISCLOSURE}

P. Valentino received travel funding and/or speaker honoraria from Biogen Idec, Merck Serono, and Euroimmun. F. Marnetto received travel funding and/or speaker honoraria from Biogen Idec, Merck Serono, and Euroimmun Italia, and received research support from Fondazione Italiana Sclerosi Multipla. L. Granieri received travel funding and/or speaker honoraria from Biogen Idec, Merck Serono, and Euroimmun. M. Capobianco served on the scientific advisory board for Biogen, Sanofi-Genzyme, Novartis, and Bayer-Schering; and received speaker honoraria from Almirall, Biogen, Novartis, Sanofi-Genzyme, and Teva. A. Bertolotto served on the scientific advisory board for Almirall, Bayer, Biogen Idec, and Genzyme; received travel funding and/or speaker honoraria from Biogen Idec, Genzyme, Novartis, Sanofi-Aventis, and Teva; served on the editorial board for Multiple Sclerosis International, Progress in Neuroscience, Dataset Papers in Neuroscience, Journal of Multiple Sclerosis, Neurology and Therapy, and Multiple Sclerosis and Demyelinating Disorders; and received research support from Merck Serono Italy, Biogen, Euroimmun, Italian Ministry of Health, Italian Multiple Sclerosis Foundation, Ricerca Biogmedica ONLUS Foundation, and San Luigi ONLUS. Go to Neurology.org/nn for full disclosure forms.

Received June 29, 2016. Accepted in final form November 1, 2016. 


\section{REFERENCES}

1. Wingerchuk DM, Hogancamp WF, O'Brien PC, Weinshenker BG. The clinical course of neuromyelitis optica (Devic's syndrome). Neurology 1999;53:1107-1114.

2. Wingerchuk DM, Lennon VA, Pittock SJ, Lucchinetti CF, Weinshenker BG. Revised diagnostic criteria for neuromyelitis optica. Neurology 2006;66:1485-1489.

3. Lennon VA, Wingerchuk DM, Kryzer TJ, et al. A serum autoantibody marker of neuromyelitis optica: distinction from multiple sclerosis. Lancet 2004;364:2106-2112.

4. Lennon VA, Kryzer TJ, Pittock SJ, Verkman AS, Hinson SR. IgG marker of optic-spinal multiple sclerosis binds to the aquaporin-4 water channel. J Exp Med 2005;202: 473-477.

5. Papadopoulos MC, Verkman AS. Aquaporin 4 and neuromyelitis optica. Lancet Neurol 2012;11:535-544.

6. Matiello M, Lennon VA, Jacob A, et al. NMO-IgG predicts the outcome of recurrent optic neuritis. Neurology 2008;70:2197-2200.

7. Weinshenker BG, Wingerchuk DM, Vukusic S, et al. Neuromyelitis optica IgG predicts relapse after longitudinally extensive transverse myelitis. Ann Neurol 2006;59: 566-569.

8. Chanson JB, Alame M, Collongues N, et al. Evaluation of clinical interest of anti-aquaporin-4 autoantibody followup in neuromyelitis optica. Clin Dev Immunol 2013;2013: 146219.

9. Waters J, Pittock SJ, Bennet JL, Jarius S, Weinshenker BG, Wingerchuk DM. Evaluation of aquaporin-4 antibody assays. Clin Exp Neuroimmunol 2014;5:290-303.

10. Kim SH, Kim W, Li XF, Jung IJ, Kim HJ. Repeated treatment with rituximab based on the assessment of peripheral circulating memory $\mathrm{B}$ cells in patients with relapsing neuromyelitis optica over 2 years. Arch Neurol 2011;68:1412-1420.

11. Pellkofer HL, Krumbholz M, Berthele A, et al. Long-term follow-up of patients with neuromyelitis optica after repeated therapy with rituximab. Neurology 2011;76: 1310-1315

12. Takahashi T, Fujihara K, Nakashima I, et al. Establishment of a new sensitive assay for anti-human aquaporin- 4 antibody in neuromyelitis optica. Tohoku J Exp Med 2006;210:307-313.

13. Takahashi T, Fujihara K, Nakashima I, et al. Antiaquaporin- 4 antibody is involved in the pathogenesis of NMO: a study on antibody titre. Brain 2007;130: $1235-1243$.

14. Jarius S, Aboul-Enein F, Waters P, et al. Antibody to aquaporin- 4 in the long-term course of neuromyelitis optica. Brain 2008;131:3072-3080.

15. Jarius $S$, Franciotta D, Paul F, et al. Testing for antibodies to human aquaporin- 4 by ELISA: sensitivity, specificity, and direct comparison with immunohistochemistry. J Neurol Sci 2012;320:32-37.

16. Isobe N, Yonekawa T, Matsushita T, et al. Quantitative assays for anti-aquaporin- 4 antibody with subclass analysis in neuromyelitis optica. Mult Scler 2012;18:1541-1551.

17. Kim W, Lee JE, Li XF, et al. Quantitative measurement of anti-aquaporin- 4 antibodies by enzyme-linked immunosorbent assay using purified recombinant human aquaporin-4. Mult Scler 2012;18:578-586.

18. Vodopivec I, Matiello M, Prasad S. Treatment of neuromyelitis optica. Curr Opin Ophthalmol 2015;26:476483.

19. Bedi GS, Brown AD, Delgado SR, Usmani N, Lam BL, Sheremata WA. Impact of rituximab on relapse rate and disability in neuromyelitis optica. Mult Scler 2011;17: 1225-1230.

20. Collongues N, de Seze J. An update on the evidence for the efficacy and safety of rituximab in the management of neuromyelitis optica. Ther Adv Neurol Disord 2016;9: 180-188.

21. Capobianco M, Malucchi S, Di Sapio A, et al. Variable responses to rituximab treatment in neuromyelitis optica (Devic's disease). Neurol Sci 2007;28:209-211.

22. Greenberg BM, Graves D, Remington G, et al. Rituximab dosing and monitoring strategies in neuromyelitis optica patients: creating strategies for therapeutic success. Mult Scler 2012;18:1022-1026.

23. Marnetto F, Granieri L, Valentino P, Capobianco M, Pautasso M, Bertolotto A. CD19 mRNA quantification improves rituximab treatment-to-target approach: a proof of concept study. J Neuroimmunol 2014;277:127-133.

24. Granieri L, Marnetto F, Valentino P, et al. Evaluation of a multiparametric immunofluorescence assay for standardization of neuromyelitis optica serology. PLoS One 2012; 7:e38896.

25. Landis JR, Koch GG. The measurement of observer agreement for categorical data. Biometrics 1977;33:159-174.

26. Kundel HL, Polansky M. Measurement of observer agreement. Radiology 2003;228:303-308.

27. Ratelade J, Verkman A. Neuromyelitis optica: aquaporin-4 based pathogenesis mechanisms and new therapies. Int J Biochem Cell Biol 2012;44:1519-1530.

28. Jarius S, Wildemann B. AQP4 antibodies in neuromyelitis optica: diagnostic and pathogenetic relevance. Nat Rev Neurol 2010;6:383-392.

29. Jarius S, Paul F, Franciotta D, et al. Mechanisms of disease: aquaporin- 4 antibodies in neuromyelitis optica. Nat Clin Pract Neurol 2008;4:202-214.

30. Kovacs KT, Kalluri SR, Boza-Serrano A, et al. Change in autoantibody and cytokine responses during the evolution of neuromyelitis optica in patients with systemic lupus erythematosus: a preliminary study. Mult Scler 2016;22: 1192-1201.

31. Nishiyama S, Ito T, Misu T, et al. A case of NMO seropositive for aquaporin- 4 antibody more than 10 years before onset. Neurology 2009;72:1960-1961.

32. Melamed E, Levy M, Waters PJ, et al. Update on biomarkers in neuromyelitis optica. Neurol Neuroimmunol Neuroinflamm 2015;2:e134. doi: 10.1212/NXI.0000000000000317.

33. Makatsori M, Kiani-Alikhan S, Manson AL, et al. Hypogammaglobulinaemia after rituximab treatment-incidence and outcomes. QJM 2014;107:821-828.

34. Roberts DM, Jones RB, Smith RM, et al. Rituximabassociated hypogammaglobulinemia: incidence, predictors and outcomes in patients with multi-system autoimmune disease. J Autoimmun 2015;57:60-65. 


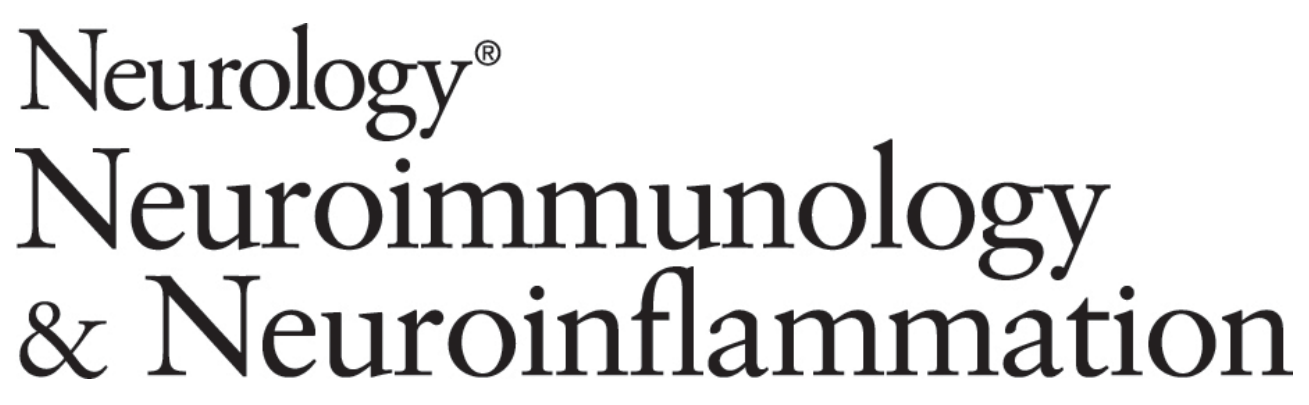

\author{
Aquaporin-4 antibody titration in NMO patients treated with rituximab: A \\ retrospective study \\ Paola Valentino, Fabiana Marnetto, Letizia Granieri, et al. \\ Neurol Neuroimmunol Neuroinflamm 2017;4; \\ DOI 10.1212/NXI.0000000000000317
}

This information is current as of December 15, 2016

Neurol Neuroimmunol Neuroinflamm is an official journal of the American Academy of Neurology.

Published since April 2014, it is an open-access, online-only, continuous publication journal. Copyright

Copyright $\odot 2016$ The Author(s). Published by Wolters Kluwer Health, Inc. on behalf of the American

Academy of Neurology. All rights reserved. Online ISSN: 2332-7812.

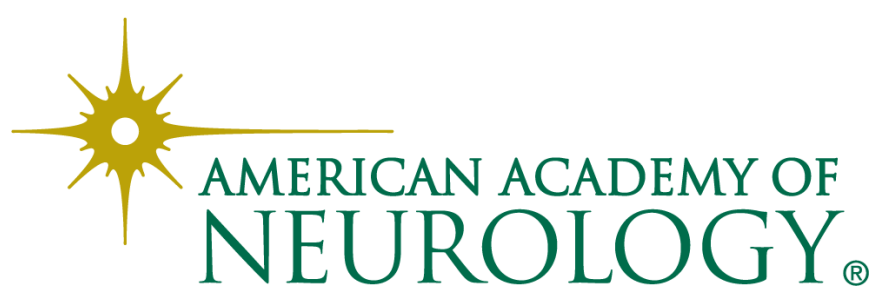




\section{Updated Information \& Services}

\section{Supplementary Material}

\section{References}

Citations

Subspecialty Collections

Permissions \& Licensing

Reprints including high resolution figures, can be found at: http://nn.neurology.org/content/4/2/e317.full.html

Supplementary material can be found at: http://nn.neurology.org/content/suppl/2016/12/15/4.2.e317.DC1

This article cites 34 articles, 1 of which you can access for free at: http://nn.neurology.org/content/4/2/e317.full.html\#\#ref-list-1

This article has been cited by 1 HighWire-hosted articles: http://nn.neurology.org/content/4/2/e317.full.html\#\#otherarticles

This article, along with others on similar topics, appears in the following collection(s):

Autoimmune diseases

http://nn.neurology.org//cgi/collection/autoimmune_diseases

Devic's syndrome

http://nn.neurology.org//cgi/collection/devics_syndrome

Diagnostic test assessment

http://nn.neurology.org//cgi/collection/diagnostic_test_assessment_

Information about reproducing this article in parts (figures,tables) or in its entirety can be found online at:

http://nn.neurology.org/misc/about.xhtml\#permissions

Information about ordering reprints can be found online:

http://nn.neurology.org/misc/addir.xhtml\#reprintsus

Neurol Neuroimmunol Neuroinflamm is an official journal of the American Academy of Neurology.

Published since April 2014, it is an open-access, online-only, continuous publication journal. Copyright

Copyright $\odot 2016$ The Author(s). Published by Wolters Kluwer Health, Inc. on behalf of the American Academy of Neurology. All rights reserved. Online ISSN: 2332-7812.

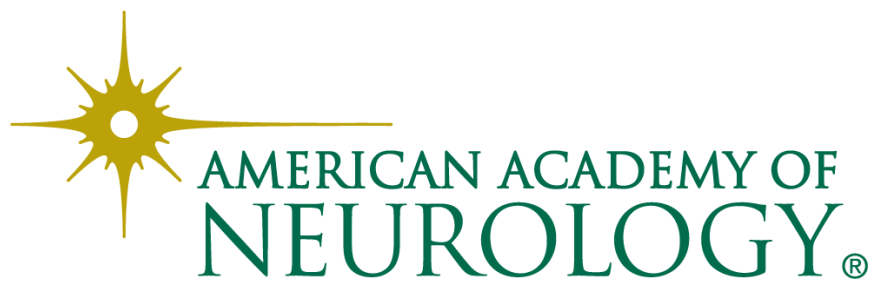

